\title{
Evaluation of clinical efficacy and safety of a newly developed automated \\ SNUH \\ cardiopulmonary resuscitation device; a porcine cardiac arrest model
} SEOUL NATIONAL UNIVERSIIY
Ki Hong Kim MD, Sang Do Shin MD, Tae Han Kim MD, Ki Jeong Hong MD, Sung Wook Choi PhD

Department of Emergency Medicine, Seoul National University Hospital

Department of Bioengineering, Kangwon National University Hospital

\section{Backgjound}

- Various automated compression device (ACD) have been adopted by emergency medical services (EMS) worldwide to ensure cardiopulmonary resuscitation (CPR) quality

- We developed a new ACD with a manual adjustment function according to compression depth and rate

- This study aimed to compare the CPR quality and chest injury between the new ACD and a conventional device (LUCAS, Physio-Control, Sweden)

\section{Methods}

- A Randomized Experimental Trial using each pigs for ACD and LUCAS group were performed to compare the CPR quality, outcomes and safety

- After 1-minute of untreated electrical-induced ventricular fibrillation, followed by 8-minutes of basic life support, and 20-minutes of advanced cardiac life support including epinephrine infusion were provided under mechanical ventilation

$$
\text { Surgical preparation }
$$
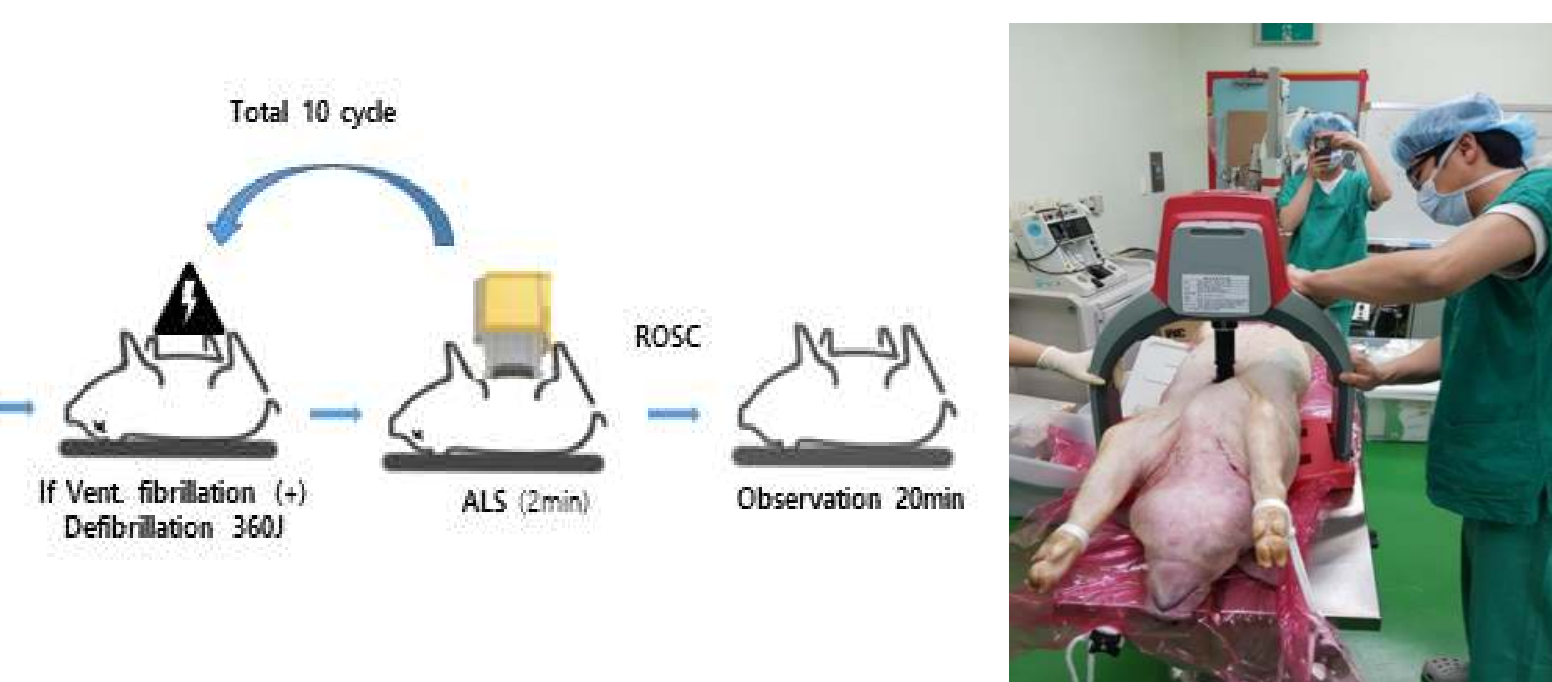

- Hemodynamic parameters were obtained and observed end-tidal CO2 (ETCO2) were continuously measured from endotracheal tube as study outcome

- Mixed model analysis and repeated measure ANOVA were used to compare the difference of hemodynamic parameters and observed ETCO2

- We examined all pigs for gross pathologic findings (number of lung contusion, cardiac contusion and tamponade) after CPR procedures through open thoracotomy

- Scoring: $(1,2,3)$ for hemorrhage up to $(1,2,3) / 3$ of surface of each lung lobe, 4 for cardiac contusion or tamponade

Conclusion

- Hemodynamic parameters and observed ETCO2 were slightly better in LUCAS group than new ACD group, but the statistical significances were not found in the porcine experimental mechanical models

\section{Results}

- There was no significant difference for hemodynamic parameters

\begin{tabular}{cccccc}
\hline \multirow{2}{*}{$\begin{array}{c}\text { hemodynamic } \\
\text { parameter }\end{array}$} & mean & median(q1-q3) & mean & median(q1-q3) & p- \\
\cline { 2 - 5 } value \\
\hline SBP & 73.96 & $73(57.3-90.5)$ & 81.98 & $78.96(59-99.11)$ & 0.39 \\
DBP & 23.49 & $23(12-33.02)$ & 13.18 & $12(7-18.84)$ & 0.26 \\
MAP & 35.27 & $36(23-45)$ & 29.39 & $30(17-39)$ & 0.71 \\
CBF & 66.90 & $65.73(42.5-82)$ & 73.58 & $63.58(42.04-101.8)$ & 0.56 \\
RAP & 32.01 & $30.8(23.99-38.59)$ & 38.92 & $31.64(20.44-56)$ & 0.61 \\
COPP & 18.78 & $18.3(13.82-22.22)$ & 15.87 & $15.14(12.42-18.48)$ & 0.41 \\
ETCO2 & 26.75 & $26(22.16-31.6)$ & 19.07 & $18(11.35-26)$ & 0.24 \\
\hline
\end{tabular}

- Repeated measure ANOVA for observed ETCO2 did not show significant difference

Model 1: All pigs during BLS phase

Model 2: sensitivity analysis for non-ROSC group
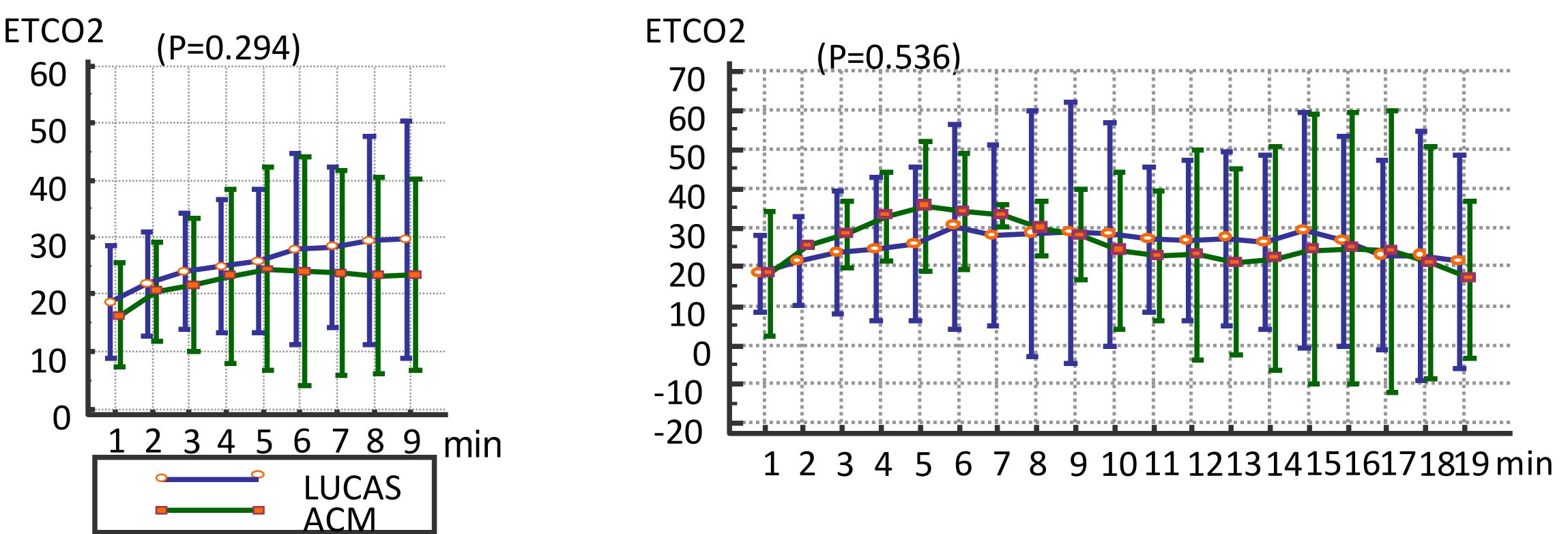

- Study subjects recovered from arrest: new ACD 5/8 vs. LUCAS 5/8

BLS survival: 5 in new ACD group / 2 in LUCAS group

- The gross pathologic findings through open thoracotomy examination after CPR showed insignificant difference of injury grade in chest cavity and wall
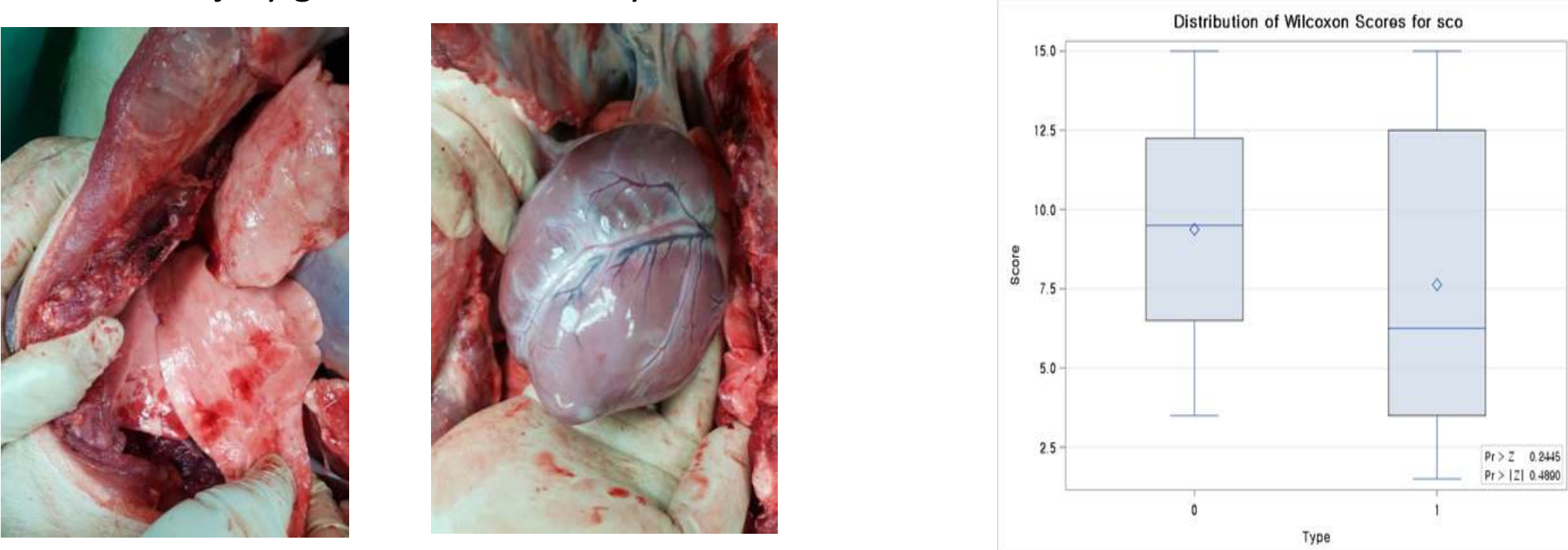

Copyright (@) 2018 Ki Hong Kim MD, emphysiciankkh@gmail.com 